\title{
ANÁLISE DA TENDÊNCIA TEMPORAL DA PRECIPITAÇÃO DO MUNICÍPIO DE FORMIGA - MG
}

\author{
Júnio da Silva Berigo ${ }^{1}$, Michael Silveira Thebaldi² \\ ${ }^{1}$ Engenheiro Ambiental e Sanitarista, Centro Universitário de Formiga - MG, Formiga \\ - MG, Brasil. \\ ${ }^{2}$ Engenheiro Agrícola, Professor Doutor do Centro Universitário de Formiga - MG, \\ Setor de Engenharia Ambiental e Sanitária (msthebaldi@uniformg.edu.br)
}

\section{Recebido em: 08/04/2017 - Aprovado em: 10/06/2017 - Publicado em: 20/06/2017 DOI: 10.18677/EnciBio_2017A5}

\begin{abstract}
RESUMO
O conhecimento da tendência temporal da precipitação é de extrema importância, pois possibilita o dimensionamento seguro de sistemas de drenagens, barragens, vertedores, sistemas de irrigação, além de possibilitar o estudo do comportamento hidrológico do local avaliado. Assim, o presente trabalho teve como objetivo analisar, por meio do teste de Mann-Kendall e de regressões lineares simples, a tendência temporal da precipitação máxima diária anual, mensal e total anual do município de Formiga - MG. Para realização do estudo, foram utilizados os dados brutos da série histórica de precipitações do município, no período de 1985 a 2015, obtidos no serviço Hidroweb da Agência Nacional de Águas. Por meio de regressão linear simples verificou-se que não houve tendência nos dados de precipitação em função do tempo, já que foram obtidos, para todas as séries, baixos coeficientes de determinação. O teste de Mann-Kendall mostrou que apenas o mês de abril apresentou discreta tendência na redução da quantidade de precipitação, porém, esta não foi significativa estatisticamente. De acordo com os resultados obtidos por meio da análise das séries de precipitação máxima diária anual, precipitação mensal e total anual, registradas no período analisado, conclui-se que não houve tendência temporal significativa, tanto relativo ao seu aumento, quanto à sua redução.
\end{abstract}

PALAVRAS-CHAVE: Gestão de Recursos Hídricos. Precipitação Máxima Diária Anual. Teste de Mann-Kendall.

\section{RAINFALL TEMPORAL TREND ANALYSIS OF FORMIGA CITY - MG}

\begin{abstract}
The knowledge of the temporal tendency of precipitation has extreme importance, because allows the safe design of drainage systems, dams, spillways, irrigation systems, besides allows the study of the hydrological behavior of the evaluated site. Thus, the objective of this study was to analyze the temporal trend of annual maximum daily, monthly and total annual rainfall of Formiga, MG, using the MannKendall test and simple linear regressions. For the study, the rainfall time series raw data of the city, from 1985 to 2015, obtained from the Hidroweb service of the Agência Nacional de Águas were used. Using the simple linear regression, it was verified that there was no trend in the rainfall data as a function of time, since for all the series, low coefficients of determination were obtained. The Mann-Kendell test showed that only the month of April showed a slight tendency to reduce the amount
\end{abstract}


of precipitation, but this was not statistically significant. According to the results obtained by the analysis of the series of annual maximum daily, monthly and total annual rainfall recorded during the analyzed period, it has been concluded that there was no significant temporal trend, both relative to its increase and its reduction.

KEYWORDS: Water Resources Management. Annual Maximum Daily Rainfall. Mann-Kendall test.

\section{INTRODUÇÃO}

A precipitação é caracterizada como o elemento mais importante do ciclo hidrológico, sendo a variável climática de maior variabilidade temporal e espacial (ALMEIDA et al., 2014). Desta forma, o conhecimento e o entendimento de alterações climáticas em escala local ou global apresenta relevância em vários ramos da pesquisa, em especial, os que tratam dos regimes de precipitação (KOSTOPOULO \& JONES, 2005).

Sobre as mudanças climáticas e os impactos associados por estas mudanças, segundo PINHEIRO et al. (2013), o clima do planeta sempre esteve sujeito a alterações em sua dinâmica, discretizadas por ciclos longos ou curtos, registrados na história. Em contrapartida, FERRARI et al. (2012) afirmam que o aquecimento global promove diretamente a alteração da variabilidade espacial e temporal das chuvas.

De acordo com JACOBI (2014) as ações antrópicas influenciam diretamente no aquecimento global e consequentemente, desencadeiam uma série de efeitos adversos, como aumento da temperatura, alteração da precipitação em várias regiões do globo, sendo que esta última afeta diretamente o manejo da água, a agricultura, energia e atividades socioeconômicas.

Desta maneira, o estudo da tendência temporal da precipitação é fundamental, pois analisando as séries históricas, é possível verificar o comportamento da precipitação, auxiliando na gestão de recursos hídricos. Neste contexto, o presente trabalho teve como objetivo analisar a tendência temporal das séries de precipitação máxima diária anual, totais mensais e anual do município de Formiga, no estado de Minas Gerais, por meio de regressão linear simples e aplicando o teste de tendência temporal de Mann-Kendall.

\section{MATERIAL E MÉTODOS}

Localizado no Centro Oeste de Minas Gerais o município de Formiga é constituído pelos biomas Cerrado e Mata Atlântica (IBGE, 2015). De acordo com a Classificação Climática de Köppen-Geiger se enquadra no clima Aw, ou seja, clima tropical de savana (Cerrado) com estação seca no inverno e verão quente, (REBOITA et al., 2015).

As séries de dados de chuva utilizados neste estudo foram obtidas junto a Agência Nacional de Águas (ANA), a partir do sistema de informações hidrológicas, o Hidroweb. O período analisado foi de 1985 a 2015 e a consulta foi realizada com o objetivo de obterem-se, após tratamento prévio dos dados, séries históricas de precipitação máxima diária anual (PMDA), precipitação total mensal e anual. Os anos de 1989 e 2006 não foram utilizados no estudo, pois estes não possuem série de dados completos.

\section{Análise de tendência temporal das séries de precipitação}

Para análise da tendência temporal dos dados de precipitação, foi utilizado o teste de Mann Kendall (MK). Dada uma série $(X 1, X 2, \ldots, X n)$ que foi extraída de 
uma amostra de inúmeras variáveis aleatórias, que são independentes e distribuidas de forma idêntica, a estatística do teste MK é representada pela Equação 1 (WAGESHO et al., 2012; DELGADO \& SOUZA, 2014):

$\mathrm{S}=\sum_{\mathrm{i}=1}^{\mathrm{n}-1} \sum_{\mathrm{j}=\mathrm{i}+1}^{\mathrm{n}} \operatorname{sinal}\left(\mathrm{X}_{\mathrm{j}}-\mathrm{X}_{\mathrm{i}}\right)$

Onde $X_{i}$ representa os valores da série, de maneira geral tomados em intervalos de tempo anuais, i e j correspondem aos índices de tempo, e $\mathrm{n}$ é o número de elementos da série. $O$ termo sinal $\left(X_{j}-X_{i}\right)$ é representado pela Equação 2:

$\operatorname{sinal}\left(X_{j}-X_{i}\right)=\left\{\begin{array}{l}+1 \text { se }\left(X_{j}-X_{i}\right)>0 \\ 0 \quad s e\left(X_{j}-X_{i}\right)=0 \\ -1 \quad s e\left(X_{j}-X_{i}\right)<0\end{array}\right.$

MANN (1945) e KENDALL (1975) afirmam que a estatística $S$ segue aproximadamente uma distribuição normal para $n \geq 8$. No caso de dados que não possuem elementos vinculados (valores iguais), a estatística de teste apresenta média $E(S)$ e variância $\operatorname{Var}(S)$ dadas, respectivamente, por:

$\mathrm{E}(\mathrm{S})=0$

$\operatorname{Var}(S)=1 / 18[n(n-1)(2 n+5)]$

$\mathrm{Na}$ ocorrência de pontos vinculados, a variância é corrigida pela expressão a seguir:

$\operatorname{Var}(S)=1 / 18\left[\mathrm{n}(\mathrm{n}-1)(2 \mathrm{n}+5)-\sum_{\mathrm{p}=1}^{q} \mathrm{t}_{\mathrm{p}}\left(\mathrm{t}_{\mathrm{p}}-1\right)\left(2 \mathrm{t}_{\mathrm{p}}+5\right)\right]$

na qual ${ }^{\mathrm{t}} \mathrm{p}$ denota o número de pontos vinculados de índices ${ }^{\mathrm{p}}$ até ${ }^{\mathrm{q}}$.

Para verificar a significânica do teste MK é utilizado um teste bilateral, com estatística padronizada $\tau$, na qual é expresso por:

$$
\tau= \begin{cases}\frac{\mathrm{S}-1}{\sqrt{\operatorname{Var}(\mathrm{S})}} & \text { se } \mathrm{S}>0 \\ 0 & \text { se } \mathrm{S}=0 \\ \frac{\mathrm{s}+1}{\sqrt{\operatorname{Var}(\mathrm{S})}} & \text { se } \mathrm{S}<0\end{cases}
$$

A hipótese nula $\left(\mathrm{H}_{0}\right)$, nos casos de ausência de tendência na série analisada, é rejeitada caso ${ }^{|\tau|>} Z_{\alpha / 2}$, onde ${ }^{\alpha}$ é o nível de significância adotado e $Z_{\alpha / 2}$ é o valor da 
distribuição normal padrão com probabilidade de excedência de ${ }^{\alpha / 2}$. $O$ sinal de T indica se a tendência é crescente $(\tau>0)$ ou decrescente $(\tau<0)$. O nível de significância normalmente utilizado é $\alpha=0,05$, no presente estudo foi seguida esta metodologia.

O p-valor da estatística S para os dados amostrados pode ser projetado pela probabilidade acumulada da distribuição normal, considerando que se $p \leq \alpha$, o teste apresenta evidências para rejeição de $\mathrm{H}_{0}$, sugerindo a presença de tendências na série. O teste de Mann-Kendall foi aplicado, utilizando o software estatístico XLStat, na versão de teste.

\section{RESULTADOS E DISCUSSÃO}

Os valores mínimos e máximos de precipitação, bem como a média e o desvio padrão das séries históricas analisadas do município de Formiga - MG são mostrados na Tabela 1.

TABELA 1. Estatística descritiva das séries de precipitação analisadas para o município de Formiga - MG

\begin{tabular}{ccccc}
\hline Série & Mínimo & Máxima & Média & Desvio Padrão \\
\hline Janeiro & 60,0 & 605,0 & 283,6 & 146,3 \\
Fevereiro & 21,0 & 376,0 & 169,9 & 90,9 \\
Março & 40,9 & 375,8 & 161,1 & 79,1 \\
Abril & 7,4 & 177,2 & 64,9 & 38,9 \\
Maio & 0,0 & 143,2 & 40,7 & 35,1 \\
Junho & 0,0 & 93,4 & 17,2 & 21,3 \\
Julho & 0,0 & 54,7 & 13,5 & 17,9 \\
Agosto & 0,0 & 77,6 & 16,1 & 22,0 \\
Setembro & 0,0 & 152,1 & 53,2 & 39,4 \\
Outubro & 10,6 & 221,0 & 96,6 & 47,2 \\
Novembro & 87,0 & 368,8 & 173,9 & 65,7 \\
Dezembro & 89,6 & 557,8 & 289,4 & 101,8 \\
Total Anual & 693,0 & 1834,6 & 1371,1 & 269,0 \\
PMDA & 35,2 & 154,0 & 84,7 & 30,0 \\
\hline
\end{tabular}

Em que: PMDA = Precipitação máxima diária anual

Ao observar a Tabela 1, tem-se que os meses de dezembro, janeiro e novembro apresentaram as maiores médias pluviométricas para o período analisado e os meses de junho, julho e agosto são os meses que apresentam os menores índices pluviométricos médios para o mesmo período.

Os meses de junho, julho e agosto apresentaram menores desvios-padrão na precipitação registrada durante o período analisado, o que infere em menor variabilidade dos dados, enquanto os meses de janeiro e dezembro mostraram maior desvio. A maior precipitação mensal total foi registrada em janeiro, enquanto que a menor, nos meses de maio a setembro, em que, em algum momento, não houve precipitação. As regressões lineares e coeficientes de determinação das séries de precipitação relativas a janeiro, fevereiro e março, são mostrados na Figura 1. 

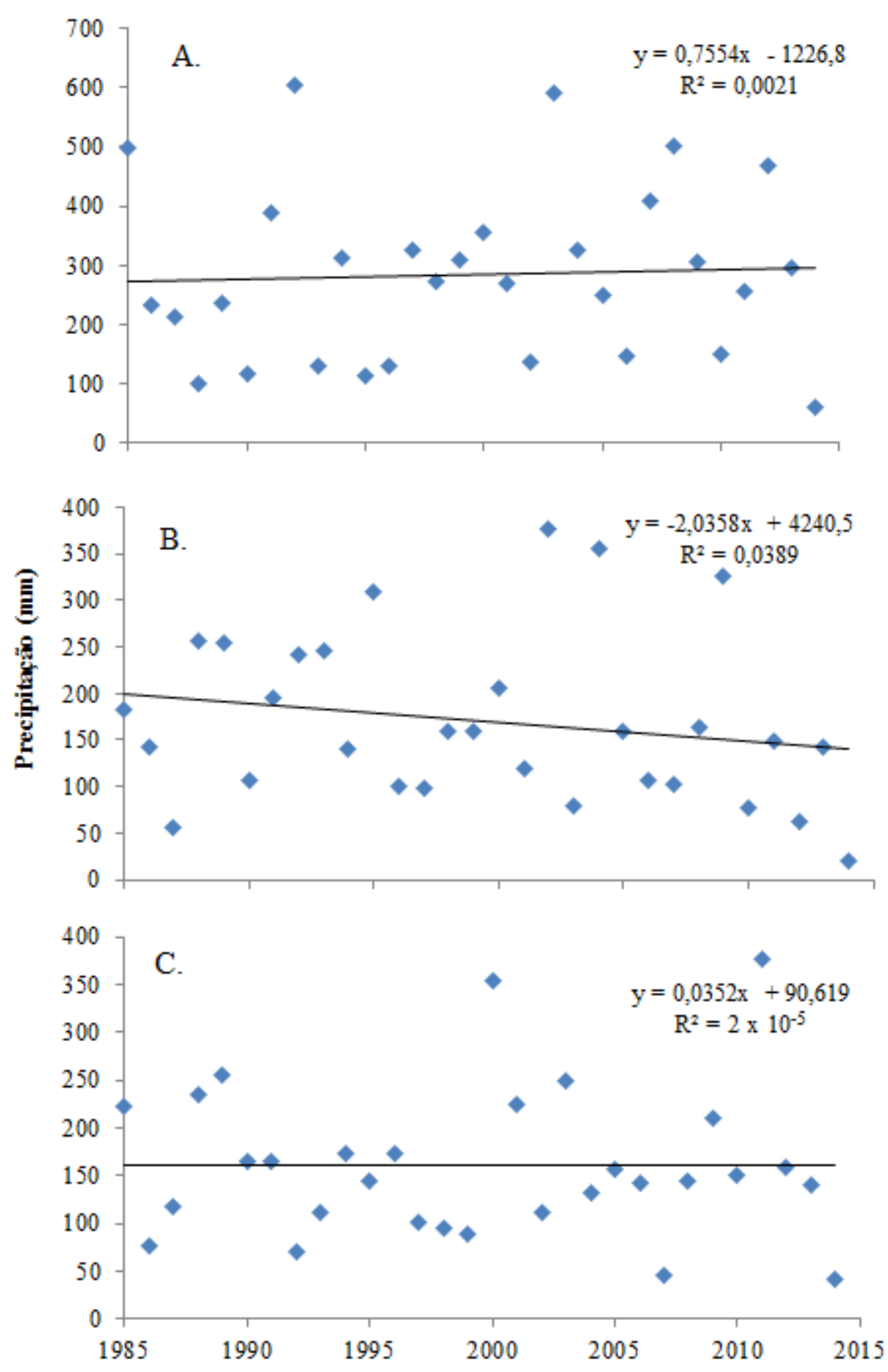

FIGURA 1. Regressão linear e coeficiente de determinação das séries de precipitação de janeiro $(A)$, fevereiro (B) e março (C). Fonte: Os autores

Os dados de precipitação referentes ao mês de março (Figura 1C) apresentam maior dispersão em relação à linha de tendência em comparação aos dados de janeiro e fevereiro (Figuras $1 \mathrm{~A}$ e $1 \mathrm{~B}$, respectivamente). Os coeficientes ENCICLOPÉDIA BIOSFERA, Centro Científico Conhecer - Goiânia, v.14 n.25; p.40 
angulares positivos das equações das retas que representam a variação da precipitação com os anos, nos meses de janeiro e março, mostram um aumento da lâmina precipitada nesses meses e o coeficiente angular negativo na reta do mês de fevereiro mostra uma queda na quantidade de precipitação registrada.

Analisando o coeficiente de determinação $\left(R^{2}\right)$ dos gráficos pode-se concluir que não houve tendência de precipitação em nenhum deles, sendo o mês de março (Figura 1C) o que apresentou o menor valor para $\mathrm{R}^{2}$, indicando, assim, a menor aderência do modelo linear em comparação aos dados observados, em relação aos meses de janeiro e fevereiro (Figuras $1 \mathrm{~A}$ e 1B, respectivamente). A regressão linear e coeficiente de determinação das séries de precipitação relativas a abril, maio e junho, são mostrados na Figura 2.

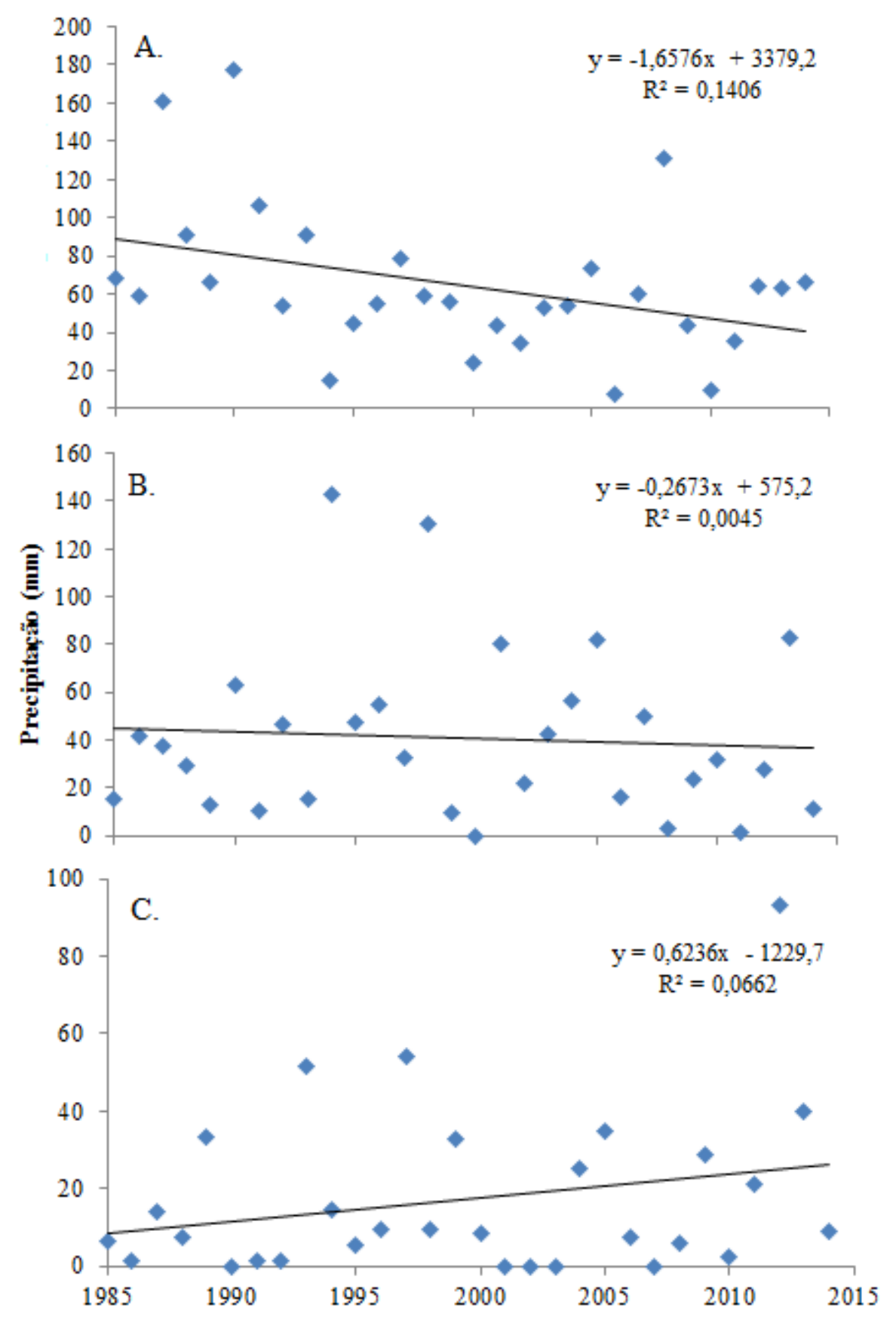


FIGURA 2. Regressão linear e coeficiente de determinação das séries de precipitação de abril (A), maio (B) e junho (C). Fonte: Os autores

O menor valor de coeficiente angular da reta foi obtido para o mês de maio (Figura 2B), o que mostra que neste obteve-se maior constância nos valores totais de precipitação registrados. Mesmo assim, o coeficiente angular deste mês é negativo, assim como para abril (Figura 2A). Já no mês de junho (Figura 2C), houve, no geral, um aumento da precipitação com o tempo. Valores diferenciados dos demais podem ser vistos para os anos de 1993 e 1997, por exemplo.

Ao analisar os valores de coeficiente de determinação, foi observado que o mês de abril (Figura 2A), dentre todos os meses avaliados, apresentou a maior concordância entre os valores observados de precipitação e o modelo linear que melhor representa estes. A regressão linear e coeficiente de determinação das séries de precipitação relativas a julho, agosto e setembro, são mostrados na Figura 3. 

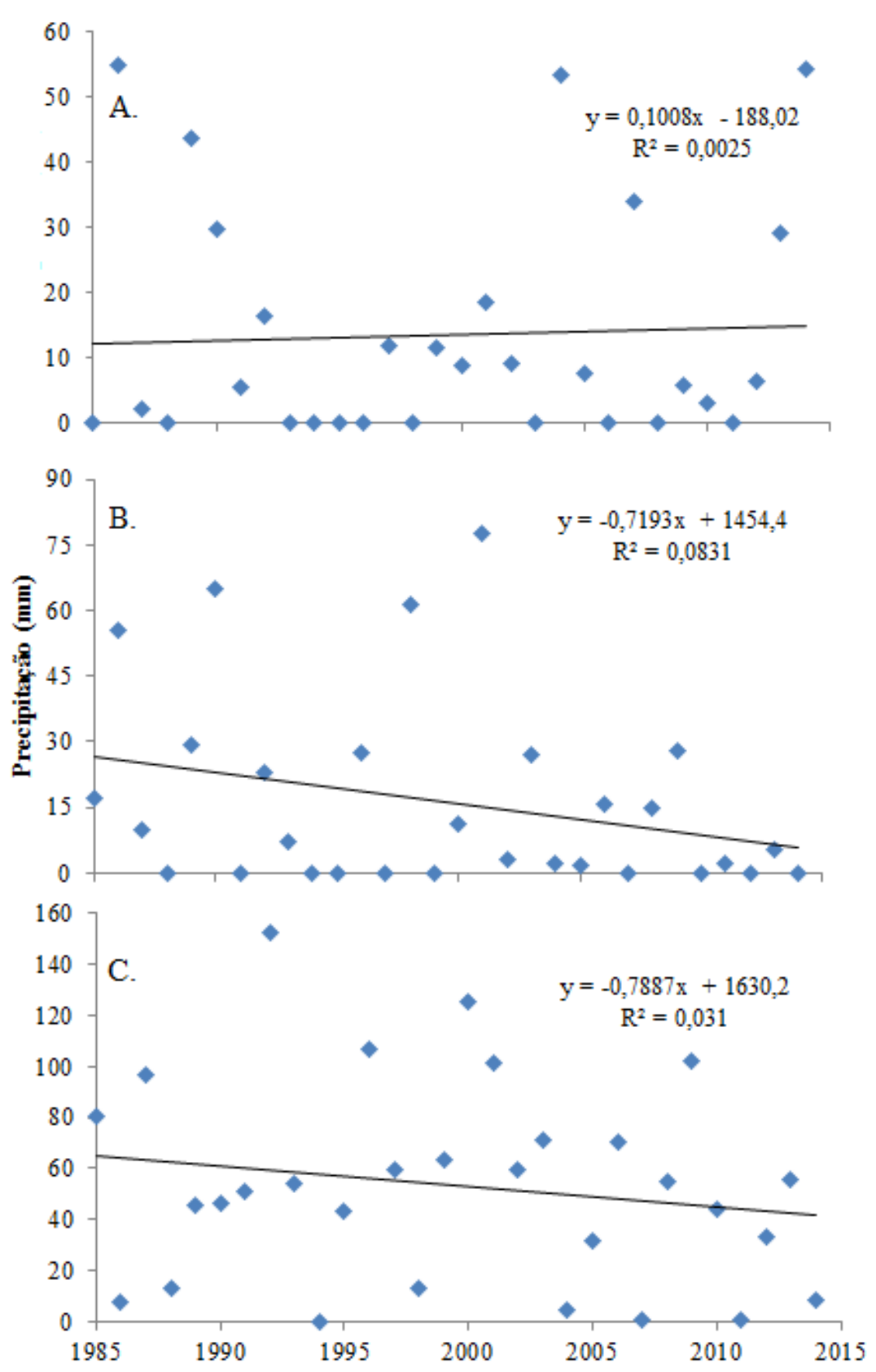

FIGURA 3. Regressão linear e coeficiente de determinação das séries de precipitação de julho $(A)$, agosto $(B)$ e setembro (C). Fonte: Os autores

De acordo com a Figura 3, os meses de julho e agosto apresentaram menores quantidades de precipitação em relação a setembro durante o período analisado. $\mathrm{O}$ mês de julho teve a menor variação geral nos valores obtidos de precipitação para estes três meses, devido ao seu valor de coeficiente angular mais próximo de zero. Os coeficientes angulares das retas nos meses de agosto e setembro indicam redução nas lâminas precipitadas. Diferentemente, o coeficiente angular da reta que melhor representa os valores de precipitação no mês de julho indica um discreto aumento na quantidade registrada no mesmo período.

O coeficiente de determinação $\left(R^{2}\right)$ dos gráficos indica baixa tendência ao aumento ou redução da precipitação neste com o passar do tempo, sendo o mês de julho (Figura $3 \mathrm{~A}$ ) o que apresentou o menor valor para $R^{2}$ entre os três. $A$ regressão 
linear e coeficiente de determinação das séries de precipitação relativas a outubro, novembro e dezembro, são mostrados na Figura 4.
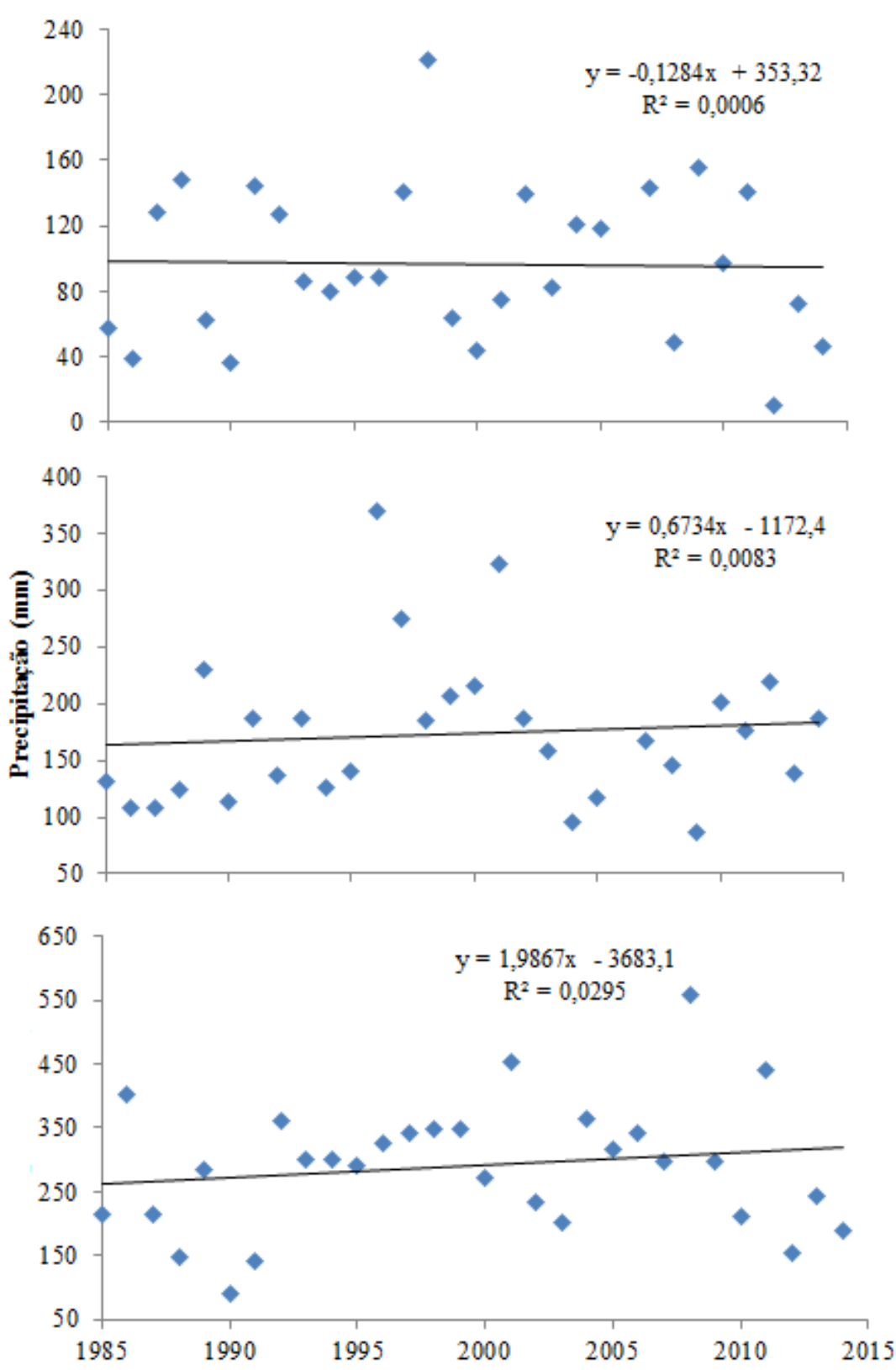

FIGURA 4. Regressão linear e coeficiente de determinação das séries de precipitação de outubro $(A)$, novembro (B) e dezembro (C). Fonte: Os autores

Pela análise da Figura 4A, tem-se uma discreta redução da precipitação total em relação ao tempo, evidenciada pelo coeficiente angular da reta que melhor representa os dados, que para o mês de outubro, possui valor negativo. Porém, este possui valor absoluto de 0,1284 , mostrando uma pequena tendência à redução. $O$ coeficiente de determinação dos valores observados em relação à reta modelada é baixo, 0,0006.

No mês de dezembro (Figura 4C), a variação da precipitação foi maior, mais acentuada que em novembro (Figura 4B), como se pode verificar pelos coeficientes angulares das retas que melhor representam as precipitações em ambos os meses com o passar dos anos. 
De acordo com a análise do último trimestre do período, o coeficiente de determinação $\left(R^{2}\right)$ dos gráficos mostra que não houve tendência de precipitação em nenhum deles, sendo o mês de dezembro (Figura 4C) o que apresentou o maior valor para $R^{2}$. A regressão linear e coeficiente de determinação das séries de precipitação máxima diária anual e precipitação total são mostradas na Figura 5.

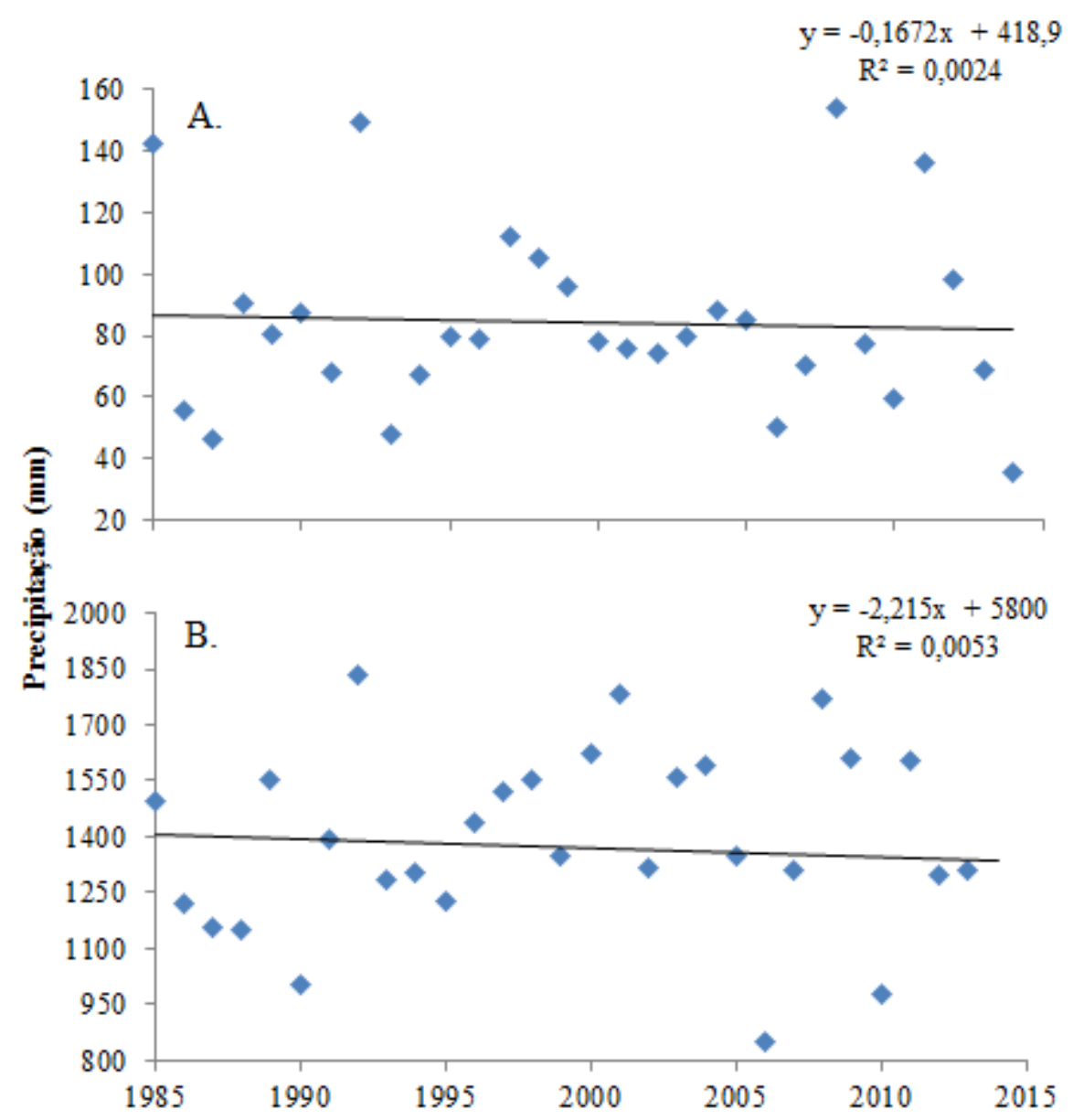

FIGURA 5. Regressão linear e coeficiente de determinação das séries de precipitação máxima diária anual (A) e precipitação total (B). Fonte: Os autores

De acordo com a Figura 5, observa-se por meio dos coeficientes angulares que houve uma redução na quantidade de precipitação máxima diária anual e total anual. Apesar da redução na quantidade de precipitação, o coeficiente de determinação dos gráficos indica baixa tendência no período analisado, sendo, respectivamente 0,0024 e 0,0053 para precipitação máxima diária anual e total anual.

Os valores referentes ao resultado do Teste de Mann - Kendall das séries históricas analisadas, do município de Formiga - MG são mostrados na tabela 2.

TABELA 2. Teste de Mann-Kendall aplicado às diferentes séries de precipitação avaliadas para o município de Formiga - MG.

\begin{tabular}{cccc}
\hline Série & $\mathrm{S}$ & $\tau$ & $\mathrm{p}$-valor \\
\hline Janeiro & 33,000 & $0,076^{\mathrm{NS}}$ & 0,572
\end{tabular}




\begin{tabular}{cccc} 
Fevereiro & $-83,000$ & $-0,191^{\mathrm{NS}}$ & 0,145 \\
Março & $-19,000$ & $-0,044^{\mathrm{NS}}$ & 0,750 \\
Abril & $-98,000$ & $-0,226^{\mathrm{NS}}$ & 0,083 \\
Maio & $-19,000$ & $-0,044^{\mathrm{NS}}$ & 0,750 \\
Junho & 54,000 & $0,126^{\mathrm{NS}}$ & 0,343 \\
Julho & 20,000 & $0,049^{\mathrm{NS}}$ & 0,728 \\
Agosto & $-69,000$ & $-0,166^{\mathrm{NS}}$ & 0,218 \\
Setembro & $-39,000$ & $-0,090^{\mathrm{NS}}$ & 0,502 \\
Outubro & $-4,000$ & $0,010^{\mathrm{NS}}$ & 0,956 \\
Novembro & 58,000 & $0,143^{\mathrm{NS}}$ & 0,288 \\
Dezembro & 30,000 & $0,069^{\mathrm{NS}}$ & 0,605 \\
Total Anual & 21,000 & $0,048^{\mathrm{NS}}$ & 0,724 \\
PMDA & $-27,000$ & $-0,062^{\mathrm{NS}}$ & 0,646 \\
\hline
\end{tabular}

Em que: $\tau=$ tendência da série; $S=$ estatística do teste

Pode-se verificar, por meio da Tabela 2, que nos meses de janeiro, junho, julho, outubro, novembro e dezembro apresentaram tendência de aumento na precipitação, verificada pelos valores positivos de $\tau$. Já para os meses de fevereiro, março, abril, maio, agosto e setembro foram verificados valores negativos de $\tau$, indicando, a priori, tendência à redução da precipitação na série analisada. Porém, em nenhuma das séries avaliadas, o valor de $\tau$ obtido, do teste de Mann-Kendall, foi significativo, como pode ser verificado pelos $p$-valores calculados para estes (Tabela 2). O mês de abril foi o que mais se aproximou de haver uma tendência significativa - mais próximo de $5 \%$ de significância estatística, apresentando o valor de $\tau$ um pvalor de 0,083.

ALMEIDA et al. (2014), ao analisarem a tendência temporal da precipitação diária máxima no estado de São Paulo, avaliaram 45 cidades utilizando séries históricas com diferentes períodos entre 1977 e 2006 e encontraram que das 45 cidades estudadas apenas cinco apresentaram tendência significativa na precipitação, portanto, a nível geral, não foi identificado tendências das precipitações diárias máximas que indicassem aumento da lâmina precipitada do estado de São Paulo, mesmo sendo identificado tendências em algumas cidades.

LIMA et al. (2011), com o uso do teste Mann-Kendall, analisaram as séries totais anuais e mensais da precipitação de 16 cidades do estado da Bahia no período de 1961 a 2009, sendo que apenas duas delas apresentaram tendências temporais significativas, enquanto que BLAIN \& MORAES (2011) realizaram o estudo das séries anuais de precipitação máxima diária das cidades de Campinas, Cordeirópolis, Mococa, Monte Alegre do Sul, Ribeirão Preto e Ubatuba no período de 1948 a 2007 e constataram que não houve tendências temporais significativas, assim como neste estudo.

Já DELGADO \& SOUZA (2014), ao analisarem a tendência da precipitação em Cruzeiro do Sul, estado do Acre concluíram, por meio de testes estatíscos aplicados a dados de precipitação, determinaram que, para este município, há aleatoriedade desta variável climatológica ao longo de 34 anos, o que infere que não houve modificação do clima local, como observado para Formiga - MG.

\section{CONCLUSÃO}

Por meio do uso de regressão linear simples e do teste Mann-Kendall para análise da tendência temporal das séries de precipitação máxima diária anual, precipitação mensal e total anual, registradas no município de Formiga - MG, entre 
1985 e 2015, conclui-se que não houve tendência temporal significativa, tanto relativo ao seu aumento, quanto à sua redução.

\section{REFERÊNCIAS}

ALMEIDA, B.M.; JOSÉ, J.V.; DUARTE, S.N.; FRIZZONE, J.A.; ARRAES, F.D.; PERBONE, A. Análise de tendência temporal da precipitação diária máxima no Estado de São Paulo. Water Resources and Irrigation Management, Campina Grande, v.3, n.1, p.1-12, 2014. Disponível em:

$<\quad$ https://www2.ufrb.edu.br/wrim/images/wrim-3-12014/WRIM_v03_n01_p_01_12.pdf>. ISSN: 2316-6886

BLAIN, G. C.; MORAES, S. O. Caracterização estatística de oito séries de precipitação pluvial máxima diária da Secretaria de Agricultura e Abastecimento do Estado de São Paulo. Revista Brasileira de Meteorologia, São José dos Campos, v.26, p.225-234, 2011. Disponível em:

< http://dx.doi.org/10.1590/S0102-77862011000200007>. doi: 10.1590/S010277862011000200007

DELGADO, R. C.; SOUZA, L. P. Tendência da precipitação e seu aproveitamento para fins não potáveis na Amazônia Ocidental, Acre. Nativa, Sinop, v. 2, n. 4, p. 108113, 2014. Disponível em: <http://dx.doi.org/10.14583/2318-7670.v02n04a04>. doi: 10.14583/2318-7670.

FERRARI, A. L.; VECCHIA, F. A. S.; COLABONE, R. O. Tendência e variabilidade anuais da temperatura e da pluviosidade em Pirassununga-SP. Revista Brasileira de Climatologia, Curitiba, v.10, n.1, p.30-46, 2012. Disponível em: < http://dx.doi.org/10.5380/abclima.v10i1.30585>. doi: /10.5380/abclima.v10i1.30585

IBGE - INSTITUTO BRASILEIRO DE GEOGRAFIA E ESTATÍSTICA . Formiga Dados gerais do município. Disponível em: $<$ http://cidades.ibge.gov.br/painel/painel.php?

lang $=\&$ codmun $=312610$ \&search=minas-gerais|formiga|infograficos:-dados-gerais-domunicipio>. Acesso em: 08 de ago. de 2016.

JACOBI, P. R. Mudanças climáticas e ensino superior: a combinação entre pesquisa e educação. Educar em Revista, Curitiba, n.3, p. 57-72, 2014. Disponível em: <http://www.scielo.br/pdf/er/nspe3/a05nspe3.pdf>. doi: 10.1590/0104-4060.38107

KENDALL, M. G. Rank Correlation Methods. London: Charles Griffin, p. 120, 1975.

KOSTOPOULO, E.; JONES, P. D. Assesment of climate extremes in the Eastern Mediterranean. Meteorology and Atmospheric Physics, Viena, v. 89, p. 69-85, 2005. Disponível em: < https://link.springer.com/article/10.1007/s00703-005-0122-2>. doi: doi:10.1007/s00703-005-0122-2

LIMA, J. R. A.; NEVES, D. J. D..; ARAÚJO, L. E.; AZEVEDO, P. V. Identificação de Tendências Climáticas no Estado da Bahia. Revista de Geografia, Recife, v. 28, n. 3 , 2011.

Disponível em: 
<http://www.revista.ufpe.br/revistageografia/index.php/revista/article/view/500/387>. ISSN: 0104-5490.

MANN, H. B. Non parametric tests against trend. Econometrica, v.13, p.245-259, 1945. Disponível em: <http://dx.doi.org/10.2307/1907187>. doi: 10.2307/1907187.

PINHEIRO, A.; GRACIANO, R. L. G.; SEVERO, D. L. Tendência das séries temporais de precipitação da região sul do Brasil. Revista Brasileira de Meteorologia, São José dos Campos, v.28, n.3, p. 281-290, 2013. Disponível em: <http://dx.doi.org/10.1590/S0102-77862013000300005>. doi: 10.1590/S010277862013000300005

REBOITA, M.S.; RODRIGUES, M.; SILVA, L.F.; ALVES, M.A. Aspectos Climáticos do Estado de Minas Gerais. Revista Brasileira de Climatologia, Curitiba, v.17, p.207-226, 2015. Disponível em: <http://dx.doi.org/10.5380/abclima.v17i0.41493>. doi: 10.5380/abclima.v17i0.41493

WAGESHO, N.; GOEL, N. K.; JAIN, M. K. Investigation of non-stationarity in hydroclimatic variables at Rift Valley lakes basin of Ethiopia. Journal of Hydrology, Amsterdam, v. 444-445, p.113-133, 2012. Disponível em:

$<$ http://cat.inist.fr/?aModele=afficheN\&cpsidt=25935865>. doi:

http://dx.doi.org/10.1016/j.jhydrol.2012.04.011. 